\title{
A perspective on vegetarian dietary patterns and risk of metabolic syndrome $e^{\text {is }}$
}

\author{
Joan Sabaté 1,2* $^{1}$ and Michelle Wien ${ }^{3}$ \\ ${ }^{1}$ Department of Nutrition, School of Public Health, Loma Linda University, 24951 North Circle Drive, Nichol Hall 1102, \\ Loma Linda, CA 92350, USA \\ ${ }^{2}$ Department of Epidemiology and Biostatistics, Loma Linda University, 24951 North Circle Drive, Nichol Hall 1102, \\ Loma Linda, CA 92350, USA \\ ${ }^{3}$ Human Nutrition and Food Science Department, California State Polytechnic University, Pomona, 3801 West Temple \\ Avenue, Pomona, CA 91768, USA
}

(Submitted 4 March 2014 - Final revision received 25 July 2014 - Accepted 18 November 2014)

\begin{abstract}
The vegetarian dietary pattern is traditionally a plant-based diet that includes fruits, vegetables, cereals, legumes, nuts, vegetable oils, soya, and possibly dairy products and/or eggs. Vegetarians and other populations who follow a plant-based dietary pattern enjoy longevity. Specifically, vegetarian dietary patterns have been associated with a lower risk for developing IHD, type 2 diabetes, hypertension, specific cancers, lower all-cause mortality and reduction in cause-specific mortality. The prevalence of the metabolic syndrome (MetS) in the USA is approximately $20 \%$ and is currently increasing in developing countries in line with the obesity epidemic. The health care costs associated with the MetS are on a magnitude of 1.6 overall compared with healthy individuals, which makes it an important public health problem. Current evidence from several cross-sectional and case-control studies shows an association between consumption of a vegetarian dietary pattern and a reduced prevalence or risk of developing the MetS. There is a need for further research to be conducted, particularly prospective cohort studies to evaluate the effect of vegetarian dietary patterns on reducing the incidence of the MetS and, clinical trials should be designed to explore vegetarian dietary patterns for the reversal of the MetS in high-risk populations. This research could contribute to reduce the societal and economic burdens associated with the disorder.
\end{abstract}

\section{Key words: Vegetarian diet: Dietary pattern: Metabolic syndrome: Mediterranean diet}

Nutritional epidemiologists encounter a multitude of challenges when conceptualising research studies that are designed to explore the beneficial effects of dietary intake on health outcomes. From a reductionist perspective, the focus is on a single nutrient, followed by foods and/or food groups. However, a healthy diet is not based on the consumption of a single nutrient or a specific food that is consumed in an isolative manner. Additionally, the synergy that exists from nutrients within and between foods can be beneficial or deleterious to specific health outcomes, and substitution effects generally occur with dietary manipulations (e.g. high intake of fibre is accompanied by a reduction in fat intake). Thus, the optimal method to evaluate diet and metabolic disorders and to promote chronic disease prevention is through a more holistic approach using dietary patterns, which has real-world applications and is

Abbreviations: AHS, Adventist Health Study; BP, blood pressure; CARDIA, Coronary Artery Risk Development in Young Adults; FBG, fasting blood glucose; HOMA, homoeostasis model of assessment; IDF, International Diabetes Foundation; IR, insulin resistance; MetS, metabolic syndrome; NCEP ATP, National Cholesterol Education Program Adult Treatment Panel; PREDIMED, Prevención con Dieta Mediterránea; WC, waist circumference.

*Corresponding author: Dr J. Sabate, fax 909558 4095, email jsabate@llu.edu

"Publication of these papers was supported by unrestricted educational grants from Federación Espanola de Sociedades de Nutrición, Alimentación y Dietética (FESNAD), International Nut and Dried Fruit Council (INC), International Union of Nutritional Sciences (IUNS), Fundación Iberoamericana de Nutrición (FINUT), Centro de Investigación Biomédica en Red de la Fisiopatología de la Obesidad y Nutrición (CIBERobn) and Centro Interuniversitario di Ricerca sulle Culture Alimentari Mediterranee (Ciiscam). The papers included in this supplement were invited by the Guest Editors and have undergone the standard journal formal review process. They may be cited. The Guest Editors declare that Salas-Salvadó is a nonpaid member of the World Forum for Nutrition Research and Dissemination of the International Nut and Dried Fruit Council. Angel Gil is President of the Fundación Iberomericana de Nutrición, which is a non-paid honorary position. Lluis Serra-Majem is the President of the Scientific Committee of the Mediterranean Diet Foundation and Scientific Director of the CIISCAM (Centro Interuniversitario di Ricerca sulle Culture Alimentari Mediterranee), Universita La Sapienza di Roma which are both non-paid, honorary positions. Goretti Guasch is the Executive Director and Member of the Executive Committee of the International Nut and Dried Fruit Council, which is a paid position. Mònica Bulló declares no conflict of interest. 
becoming the gold standard in nutritional epidemiological research.

Studies of dietary patterns generally address the amounts, proportions, frequency and variety of foods that are habitually consumed in a population and their subsequent relationship with metabolic disorders and chronic diseases. A caveat in the study of dietary patterns is that universal definitions of dietary patterns vary from study to study, which makes comparisons between studies challenging. Although many dietary patterns (e.g. Western ${ }^{(1,2)}$, healthy or prudent ${ }^{(3)}$, Mediterranean ${ }^{(4)}$ ) are associated with an increased or decreased risk for developing metabolic disorders and chronic diseases, the primary focus of the present study will be limited to presenting the existing evidence on vegetarian dietary patterns and the risk of developing the metabolic syndrome (MetS). The present paper was written based on the articles used for developing a scientific presentation titled 'Vegetarian Diets and Risk of Metabolic Syndrome - Mediterranean Diets as a Paradigm' for the 1st World Forum for Nutrition Research Conference, which was held in Reus, Spain on 21 May 2013. Also, a MEDLINE search was performed in PubMed using the MeSH terms 'metabolic syndrome' and 'vegetarian diet' in the abstract and title of the article, which yielded six additional relevant articles that were incorporated into the paper.

\section{Metabolic syndrome}

The MetS is a cluster of disorders that is associated with an increased risk for developing type 2 diabetes and $\mathrm{CVD}^{(5,6)}$. The MetS is present in $78 \%$ of persons with prediabetes and in $86 \%$ of individuals with type 2 diabetes $^{(7)}$. The prevalence of the MetS in the USA is approximately $20 \%{ }^{(8)}$ and is currently increasing in developing countries in line with the obesity epidemic ${ }^{(9)}$. The health care costs associated with the MetS are on a magnitude of 1.6 overall compared with healthy individuals ${ }^{(10)}$, which makes it an important public health problem.

Two main classification systems are in place for the clinical identification of the MetS, the 2001 National Cholesterol Education Program (NCEP) Adult Treatment Panel (ATP) III criteria, which was updated in 2005 by the American Heart Association and the National Heart Lung and Blood Institute, and the 2005 International Diabetes Foundation (IDF) criteria. The NCEP ATP III defines the MetS as the presence of three or more of the following five criteria: waist circumference (WC) $>40$ inches (male) or $>35$ inches (female); blood pressure $(\mathrm{BP})>130 / 85 \mathrm{mmHg}$; fasting $\mathrm{TAG}>1.69 \mathrm{mmol} / \mathrm{l}$; fasting $\mathrm{HDL}<1.03 \mathrm{mmol} / 1$ (male) or $1.29 \mathrm{mmol} / 1$ (female); and fasting blood glucose $(\mathrm{FBG})>5.56 \mathrm{mmol} / \mathrm{l}^{(11)}$. The IDF criteria includes the same criteria as the NCEP ATP III; however, it requires that obesity, but not insulin resistance (IR), be present ${ }^{(12)}$. Also, population-specific cutpoints for obesity exist to account for the variable distributions of norms for body weight and WC across different populations, ethnicities and nationalities. Hence, in light of the increasing prevalence of the MetS worldwide and the associated health consequences, there is a pressing need to identify dietary patterns and other lifestyle factors that may be effective in the prevention and management of the MetS.

\section{Vegetarian dietary patterns}

A vegetarian dietary pattern is operationally defined in epidemiological studies based on what is excluded from the diet (e.g. degree of animal food intake) $v$. what is consumed on a regular basis, which becomes problematic when comparing health outcomes across cohort studies. Additionally, the questions that frequently arise are "What do vegetarians eat?' and 'Do vegetarians eat healthy?' The vegetarian dietary pattern is traditionally a plant-based diet that includes fruits, vegetables, cereals, legumes, nuts, vegetable oils, soya, and possibly dairy products and/or eggs. Vegetarians ${ }^{(13,14)}$ and other populations that follow a plant-based diet ${ }^{(15)}$, plant-based low-carbohydrate diet ${ }^{(16)}$, Mediterranean diet ${ }^{(17-20)}$ and healthy or prudent diet ${ }^{(17,21,22)}$ enjoy longevity. Specifically, vegetarian dietary patterns have been associated with a lower risk for developing $\operatorname{IHD}^{(13,23)}$, type 2 diabetes $^{(24,25)}$, hypertension ${ }^{(26,27)}$, specific cancers ${ }^{(28)}$, lower all-cause mortality ${ }^{(29)}$ and reduction in cause-specific mortality $^{(29)}$.

\section{Obesity and insulin resistance}

Obesity and IR are both significant contributing factors in the development of the MetS. Consistent findings across six epidemiological studies of adult vegetarians (Adventist Mortality Study, Adventist Health Study-1 (AHS-1), AHS-2, Heidelberg Study, Oxford Vegetarian Study, EPIC-Oxford) have shown that a progressive increase in BMI is observed as meat and animal products are increasingly included in the $\operatorname{diet}^{(30)}$. Further, strict vegetarian and lacto-ovo-vegetarian diets afford greater protection against overweight than omnivores. Irrespective of age, sex, race and geography, vegetarians are leaner than non-vegetarians and have a lower risk of obesity $^{(24)}$.

In a case-control study involving 391 female Buddhist vegetarians (approximately 80\% lacto-ovo-vegetarian) and 315 female Buddhist non-vegetarians attending a health clinic in Taiwan ${ }^{(31)}$, a subgroup of non-diabetic participants who were vegetarian were found to have a $29 \%$ lower risk of homoeostasis model of assessment-IR (HOMA-IR) compared with non-vegetarians (OR $0.71,95 \%$ CI 0.48, 1.06; $P=0.094)$. Similarly, a case-control study of healthy normalweight adults (age 19-64 years) residing in Bratslava(32) found lower FBG $(P<0.01)$ and HOMA-IR $(P<0.001)$ values among ninety-five long-term lacto-Ovo-vegetarians compared with 107 non-vegetarians (e.g. Western diet). With respect to age, HOMA-IR was significantly higher in the 31- to 40-year age range and in the later age decades among the nonvegetarians compared with the lacto-ovo-vegetarians. These findings are supportive of dairy products as a component of a healthy vegetarian diet ${ }^{(33)}$ and are consistent with the evidence from the Coronary Artery Risk Development in Young Adults (CARDIA) study ${ }^{(34)}$ that found that each daily serving of dairy products reduced the risk of developing IR by $21 \%$. 
Table 1. Overview of select studies on vegetarian dietary patterns and metabolic syndrome (MetS) risk factors

\begin{tabular}{|c|c|c|c|}
\hline Authors (year) & Study design & Groups & Results \\
\hline Rizzo et al. (2011) & Cross-sectional ( $n$ 773) & $\begin{array}{l}\text { Vegetarian }(35 \%) \\
\text { Semi-vegetarian }(16 \%) \\
\text { Non-vegetarian }(49 \%)\end{array}$ & $\begin{array}{l}\text { Reference group: Non-vegetarian } \\
\text { Vegetarian: } 56 \% \text { reduced risk of MetS and lower means for TAG, } \\
\text { FBG, BP and WC. }\end{array}$ \\
\hline Yang et al. (2012) ${ }^{(36)}$ & Cross-sectional ( $n$ 295) & $\begin{array}{l}\text { Lacto-vegetarian }(57 \%) \\
\text { Non-vegetarian }(43 \%)\end{array}$ & $\begin{array}{l}\text { Reference group: Non-vegetarian } \\
\text { Lacto-vegetarian: lower means for TAG, FBG, BP, BMI and TC:HDL ratio. }\end{array}$ \\
\hline Shang et al. $(2011)^{(39)}$ & Retrospective cohort ( $n$ 93209) & $\begin{array}{l}\text { Vegan }(1 \%) \\
\text { Non-vegetarian }(91 \%) \\
\text { Lacto-vegetarian }(5 \%) \\
\text { Pesco-vegetarian }(3 \%)\end{array}$ & $\begin{array}{l}\text { Reference group: Vegan } \\
\text { Non-vegetarian: } 25 \% \text { reduced risk of MetS; } 28 \% \\
\text { reduced risk of low HDL; } 16 \% \text { higher risk of high FBG. } \\
\text { Lacto-vegetarian: } 19 \% \text { reduced risk of MetS. } \\
\text { Pesco-vegetarian: } 32 \% \text { reduced risk of MetS and } 30 \% \text { reduced risk of low HDL. }\end{array}$ \\
\hline Amini et al. $(2010)^{(37)}$ & Cross-sectional ( $n$ 425) & $\begin{array}{l}\text { Vegetarian pattern } \\
\text { Chicken and plant pattern } \\
\text { Prudent pattern } \\
\text { High-fat dairy pattern } \\
\text { Western pattern }\end{array}$ & $\begin{array}{l}\text { Reference: The first tertile of dietary pattern scores } \\
\text { Vegetarian pattern: greater odds of high FBG (OR 2.26). } \\
\text { Prudent pattern: lower odds of low HDL (OR = 0.55). } \\
\text { Western pattern: greater odds of TAG (OR 1.76) and of high BP (OR 2.62). }\end{array}$ \\
\hline Gadgil et al. $(2013)^{(38)}$ & Cross-sectional $(n$ 150) & $\begin{array}{l}\text { Vegetarian }(61 \%) \\
\text { Non-vegetarian }(39 \%)\end{array}$ & $\begin{array}{l}\text { Reference group: Non-vegetarian } \\
\text { Vegetarian: lower means for FBG and HOMA-IR. }\end{array}$ \\
\hline Huang et al. $(2011)^{(52)}$ & Cross-sectional ( $n$ 1071) & $\begin{array}{l}\text { Ovo-vegetarian or } \\
\text { lacto-ovo-vegetarian }(25 \%) \\
\text { Non-vegetarian }(75 \%)\end{array}$ & $\begin{array}{l}\text { Reference group: Ovo-vegetarian or lacto-ovo-vegetarian } \\
\text { Non-vegetarian: no difference in the risk of high BP or MetS. }\end{array}$ \\
\hline Kim \& Bae $(2012)^{(67)}$ & Cross-sectional $(n$ 107) & $\begin{array}{l}\text { Vegetarian }(55 \%) \\
\text { Non-vegetarian }(45 \%)\end{array}$ & $\begin{array}{l}\text { Reference group: Non-vegetarian } \\
\text { Vegetarian: lower means for body weight, BMI, percent body fat, WC, } \\
\text { systolic BP, diastolic BP and FBG. The prevalence of MetS tended to } \\
\text { be lower (33.9\%) compared with non-vegetarian ( } 47.9 \%) \text {. }\end{array}$ \\
\hline Chiang et al. (2013) $)^{(31)}$ & Case-control $(n 706)$ & $\begin{array}{l}\text { Vegetarian }(55 \%) \\
\text { approximately } \\
80 \% \text { Lacto-ovo } \\
\text { Non-vegetarian }(45 \%)\end{array}$ & $\begin{array}{l}\text { Reference group: Non-vegetarian } \\
\text { Vegetarian: lower means for BMI, WC, TC, LDL, HDL, TC:HDL ratio, } \\
\text { LDL:HDL ratio and HOMA-IR. } \\
\text { Lacto-ovo-vegetarian: for } 1-11 \text { years' duration, a } 54 \% \text { reduced risk of } \\
\text { MetS by IDF criteria and } 45 \% \text { reduced risk by modified NCEP criteria. } \\
\text { For }>11 \text { years' duration, a } 57 \% \text { reduced risk of MetS by IDF criteria and } 42 \% \\
\text { reduced risk by modified NCEP criteria. }\end{array}$ \\
\hline Valachovicová (2006) ${ }^{(32)}$ & Case-control ( $n$ 202) & $\begin{array}{l}\text { Lacto-ovo-vegetarian ( } 47 \%) \\
\text { Non-vegetarian }(53 \%)\end{array}$ & $\begin{array}{l}\text { Reference group: Non-vegetarian } \\
\text { Vegetarian: lower means for FBG and HOMA-IR, and HOMA-IR } \\
\quad \text { was lower in the age range of } 31-40(n 21), 41-50(n \text { 38) and } 51-64(n 16) \text { years. }\end{array}$ \\
\hline
\end{tabular}

FBG, fasting blood glucose; BP, blood pressure; WC, waist circumference; TC, total cholesterol; HOMA-IR, homoeostasis model of assessment-insulin resistance; IDF, International Diabetes Foundation; NCEP, National Cholesterol Education Program. 


\section{Cross-sectional studies}

A cross-sectional study of 773 subjects (age 30-94 years) that participated in a Calibration Sub-study of the AHS-2 was conducted to evaluate the risk of the MetS according to dietary pat$\operatorname{tern}^{(35)}$. Dietary patterns were categorised as vegetarian (meat, poultry or fish $<1$ time/month), semi-vegetarian (fish at any frequency and consuming other meat products $<1$ time/ month or total meat $\geq 1$ time/month and $<1$ time/week) and non-vegetarian (red meat or poultry $\geq 1$ time/month and total of all meat products $\geq 1$ time/week) using a validated FFQ. A vegetarian dietary pattern was associated with a $56 \%$ lower risk of having the MetS compared with a non-vegetarian dietary pattern (OR 0.44, $95 \%$ CI 0.30, 0.64; $P<0.001)$ and yielded significantly lower means for TAG, FBG, BP and WC (Table 1). The more favourable metabolic risk factor profile persisted after adjustments were made for other lifestyle (e.g. educational status, smoking, alcohol intake, physical activity and dietary energy intake) and demographic (e.g. age, sex and ethnicity) factors. Further, the prevalence of the MetS was 25.2\% among vegetarians, $37 \cdot 6 \%$ among semi-vegetarians and $39 \cdot 7 \%$ among non-vegetarians ( $P$ for trend $<0 \cdot 001$ ).

Consistent findings were observed in another crosssectional study ${ }^{(36)}$ that was conducted among 196 healthy Chinese lacto-vegetarians and 126 healthy Chinese omnivores (age 21-76 years), which found to have lower BMI, BP and serum levels of TAG, total cholesterol, LDL, FBG and the total cholesterol:HDL ratio in the lacto-vegetarian group (all $P<0.05$ ) (Table 1). Additional analysis showed that the lacto-vegetarians (age 24-55 years) had a lower probability of developing CVD in 5-10 years compared with the omnivores using the same age range criteria.

In a cross-sectional study among 425 adults (age $35-55$ years) with impaired glucose tolerance participating in the Isfahan Diabetes Prevention Study ${ }^{(37)}$, a Western diet was positively associated with high TAG (OR 1.76, 95\% CI 1.01, 3.07) and high BP (OR 2.62, 95\% CI 1.32, 5.23) whereas a prudent diet was associated with reduced odds of low HDL (OR 0.55, 95\% CI $0.31,0.96)$. Additionally, a vegetarian diet was inversely associated with elevated FBG (OR 2.26, $95 \%$ CI 1.25, 4.06). Consistent with these observations, Gadgil et al. ${ }^{(38)}$ have recently reported that among 150 Asian Indians (age 45-84 years) participating in the Metabolic Syndrome and Atherosclerosis in South Asians Living in America (MASALA) study, a vegetarian diet was associated with lower HOMA-IR $(P=0.05)$ compared with a Western diet in the context of lower HDL concentrations $(P=0 \cdot 09)$. Lower HDL concentrations have similarly been reported in the aforementioned case-control study involving the female Buddhist vegetarians in Taiwan ${ }^{(31)}$. Compared with the non-vegetarians, a reduced risk for the MetS was found for lacto-ovo-vegetarians of $1-11$ years' duration and $>11$ years' duration by $45 \%$ (OR $0.55,95 \%$ CI $0.32,0.92)$ and $42 \%$ (OR 0.58 , $95 \%$ CI 0.33, 0.997), respectively.

\section{Retrospective cohort studies}

A retrospective cohort study was conducted to assess the risk of the MetS among 93209 Taiwanese who were categorised as vegan, pesco-vegetarian, lacto-vegetarian and non-vegetarian using data from a longitudinal health check-up database ${ }^{(39)}$. After adjusting for demographic and lifestyle factors, the hazard ratios of the MetS for non-vegetarians, pesco-vegetarians and lacto-vegetarians were 0.75 (95\% CI $0.64,0.88), 0.68$ (95\% CI $0.55,0.83)$ and $0.81(95 \% \mathrm{CI} 0.67,0.97)$ compared with vegans. In terms of specific MetS risk factors, non-vegetarians and pesco-vegetarians had a 28 and $30 \%$ reduced risk of developing low HDL than vegans with an adjusted hazard ratio of 0.72 (95\% CI $0.62,0.84)$ and 0.70 (95\% CI 0.57, 0.84), respectively (Table 1). The investigators suggested that the low dietary fat intake among the vegans could have induced low HDL levels, which has been described by others comparing lowand high-fat diets on CVD risk factors ${ }^{(40,41)}$. Lastly, compared with vegans, non-vegetarians demonstrated a $1 \cdot 16$ times risk of high FBG (95\% CI 1.02, 1.32), which may be due to the effect of a higher fat intake on diminishing insulin sensitivity ${ }^{(42-46)}$.

\section{Dietary pattern analysis}

To address the complexity of dietary intake, nutritional epidemiologists have embraced dietary pattern analysis to examine the association between dietary patterns and the MetS. A 2006 review of three large robust epidemiological studies ${ }^{(47)}$, more specifically the Isle of Ely ${ }^{(48)}$, Malmo Diet and Cancer Project $^{(49)}$, and the CARDIA study ${ }^{(34)}$, has shown that dietary patterns high in fruits, vegetables and dairy products are associated with a lower prevalence of the MetS, and dietary patterns with high meat intake are associated with components of the MetS, particularly impaired glucose tolerance. Further, minimally processed cereals are associated with a decreased risk of the MetS while highly processed cereals with a high glycemic index are associated with a higher risk. These data strengthen the findings from the aforementioned large studies of vegetarians and emphasise the importance of healthy food patterns for preventing metabolic disturbances. The investigators concluded that no single dietary component could be considered wholly responsible for the association of diet with the MetS and that the overall diet quality appears to confer protection against the MetS.

More recently, a prudent dietary pattern (e.g. dairy products, whole grains, fruits, vegetables and coffee) was compared with a Western dietary pattern (e.g. meat, refined grains, fried foods and sweetened beverages) using prospective data from 9514 subjects (age 45-64 years) participating in the Atherosclerosis Risk in Communities (ARIC) Study ${ }^{(50)}$. Over 9 years of follow-up, the Western pattern was adversely associated with incident MetS ( $P$ for trend=0.03). Further analysis of individual food groups showed that meat, fried foods and diet soda were adversely associated with the MetS and dairy intake was protective $(P$ for trend $<0.05$ for all). However, no associations were found between incident MetS and a prudent dietary pattern, nor the intake of whole grains, refined grains, fruits, vegetables, nuts, coffee or sweetened beverages.

The use of factor analysis to derive dietary patterns within South Asian ${ }^{(51)}$, Taiwanese ${ }^{(52)}$ and South Korean ${ }^{(53)}$ adult populations has revealed conflicting results in the context of 
vegetarian diet patterns and the MetS risk factors. First, among South Asians living in the UK who experienced dietary acculturation $^{(51)}$, a reduction in vegetarianism and acceptance of a Western dietary pattern was not associated with a higher prevalence of the MetS. The investigators speculated that the inclusion of full-fat dairy products and low consumption of raw fruits and vegetables may partially explain their surprising findings. Second, using data from 1071 elderly participants (age $\geq 65$ years) in the Elderly Nutrition and Health Survey in Taiwan (1999-2000), Huang et al. ${ }^{(52)}$ failed to observe an association between the MetS for vegetarians compared with omnivores. Lastly, a dietary pattern that included grains, vegetables and fish was associated with a lower risk of high TAG ( $P$ for trend $<0.01$ ) and a $14 \%$ reduced risk of the MetS $(P$ for trend $=0.02$ ) among 9850 South Korean adults who participated in the 2001 and 2005 Korean National Health and Nutrition Examination Surveys ${ }^{(53)}$.

\section{Childhood dietary intake studies}

The investigative team for the Cardiovascular Risk in Young Finns Study recently explored the associations between childhood dietary factors (e.g. frequency of vegetable, fruit, fish and meat intake, and use of butter on bread) and physical activity and risk of the MetS in adulthood ${ }^{(54)}$. After 27 years of follow-up in a cohort of 2218 (age at baseline 3-18 years), a $14 \%$ decrease in the risk of developing the MetS was observed for a higher intake of childhood vegetable consumption (OR $0.86,95 \%$ CI $0.77,0.97$ ). Further, a lower intake of vegetable consumption predicted high $\mathrm{BP}$ (OR 0.88, 95\% CI $0 \cdot 80,0.98)$ and high TAG (OR 0.88, $95 \%$ CI 0.79, 0.99). These results complement our previous findings from the ChildAdolescent Blood Pressure Study that showed that the frequency of vegetables (OR 0.67, 95\% CI 0.48, 0.94), grains (OR $0 \cdot 59,95 \%$ CI $0 \cdot 41,0 \cdot 83$ ), nuts (OR $0 \cdot 60,95 \%$ CI $0 \cdot 43,0 \cdot 85)$ and low-nutrient-dense foods (OR 0.43, 95\% CI 0.29, 0.63) were inversely related to the risk of being overweight in a cohort of 1764 healthy children and adolescents (age 6-19 years) ${ }^{(55)}$

\section{Mediterranean dietary pattern}

The Mediterranean dietary pattern has many attributes that resemble a vegetarian dietary pattern (Fig. 1) because it is

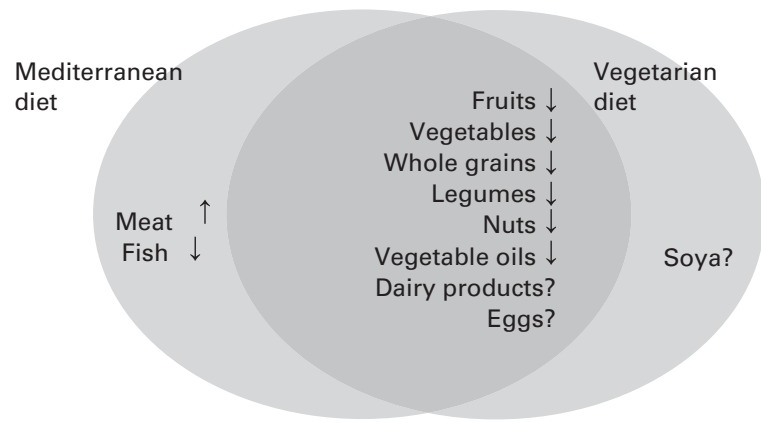

Fig. 1. Foods related to the metabolic syndrome (MetS) in two plant-based diets. $\uparrow$, increases the risk of MetS; $\downarrow$, decreases the risk of MetS; ?, uncertain relation to MetS. traditionally characterised by a high consumption of fruits, vegetables, legumes, grains, nuts and olive oil, a moderate intake of wine and dairy products, and a low intake of red and processed meat, cream and pastries. It is beyond the scope of the present paper to discuss the extensive body of published articles that describe the association between the Mediterranean diet and the MetS. However, it is worth discussing the findings from a recent meta-analysis of epidemiological studies and randomised controlled trials in addition to a cross-sectional study and the one-year results of a randomised trial among high CVD risk adults that participated in the Prevención con Dieta Mediterránea (PREDIMED) trial.

First, a meta-analysis of fifty original research studies (thirty-five clinical trials, two prospective studies and thirteen cross-sectional trials) with 534906 participants was conducted through 30 April 2010 to assess the effect of a Mediterranean diet on the MetS and its individual components ${ }^{(56)}$. Adherence to the Mediterranean diet in the prospective studies and clinical trials was found to be associated with a $31 \%$ reduced risk of the MetS. Further, results from both the epidemiological studies and the clinical trials revealed a protective role of the Mediterranean diet on WC, HDL, TAG, systolic BP, diastolic BP and FBG. Thus, the Mediterranean diet pattern is an option for the primary and secondary prevention of the MetS and its components.

The relationship between adherence to the Mediterranean dietary pattern and risk of the MetS was also evaluated among 808 participants at high CVD risk enrolled in the PREDIMED trial using a cross-sectional approach ${ }^{(57)}$. Compared with the lowest quartile of dietary adherence, participants with the highest dietary adherence had a 56, 47 and 54\% lower odds of having the MetS, low HDL and high TAG, respectively. Further, consumption of olive oil, legumes and red wine was associated with lower odds of having the MetS. Second, in the entire cohort of 1224 participants after 1 year of follow-up ${ }^{(58)}$, incident rates of the MetS were similar among the three study arms (Mediterranean diet + olive oil, Mediterranean diet + mixed nuts or a low-fat diet (control)). Compared with a $2 \%$ reduction in the MetS prevalence in the control arm, a 13.7 and $6.7 \%$ reduced prevalence was found in the mixed nut $(P=0 \cdot 01)$ and olive oil $\operatorname{arm}(P=0 \cdot 18)$, respectively. After adjustments for confounders, the OR for the reversion of the MetS were $1 \cdot 3$ (95\% CI 0.8, 2.1) and 1.7 (95\% CI $1 \cdot 1,2 \cdot 6)$ for the olive oil and mixed nuts arms compared with the control arm, respectively. The investigators concluded that a nut-enriched Mediterranean diet may be a useful tool in the management of the MetS among high CVD risk adults, which is consistent with the views of others ${ }^{(59)}$.

The novel findings from these two PREDIMED studies prompted a cross-sectional analysis of the data from the aforementioned AHS-2 Calibration Sub-study to explore the relationship between total nut (tree nuts and peanuts) consumption, the MetS and obesity ${ }^{(60)}$. In addition to finding the odds of the MetS and obesity to be inversely related to the amount, frequency and the pattern of nuts consumed, WC was $40 \%$ lower among high-tree nut consumers (mean intake of $16 \mathrm{~g} / \mathrm{d})$ compared with low-tree nut consumers (mean intake of $5 \mathrm{~g} / \mathrm{d}$ ) (OR 0.60, 95\% CI 0.39, 0.93). Complementary to the AHS-2 Calibration Sub-study and PREDIMED 
findings, a study that evaluated out-of-hand nut consumers (operationally defined as consuming $\geq 1 / 4$ ounce of nuts/d) among the 13292 adults (age $>19$ years) participating in the United States National Health and Nutrition Examination Survey (1999-2004) observed that the nut consumers had a higher HDL $(P<0 \cdot 01)$ and a $19 \%$ decreased risk of high BP (OR 0.81, 95\% CI 0.67, 0.98) compared with non-consumers, which are two MetS risk factors ${ }^{(61)}$

\section{Potential mechanisms}

Dietary patterns (e.g. vegetarian, Mediterranean and prudent) that feature a high consumption of fruits and vegetables may reduce the risk of the MetS through mechanisms that rely on a beneficial synergistic combination of antioxidants, fibre, $\mathrm{K}, \mathrm{Mg}$ and phytochemicals, which may result in a decrease in BMI, BP, TAG, oxidative stress, IR and systemic inflammation. Evidence for the latter hypothesis is supported by a cross-sectional study of 486 Tehrani female teachers (age $40-60$ years) ${ }^{(62)}$ that found an inverse association with plasma C-reactive protein concentrations and fruit and vegetable intakes using a validated semiquantitative FFQ. Participants in the highest quintile of fruit intake and vegetable intake had a 34 and $30 \%$ reduced chance of having the MetS than those in the lowest quintiles, respectively.

Omnivores consume heme iron from animal products and have significantly higher concentrations of serum ferritin compared with vegetarians ${ }^{(63-65)}$. Evidence has emerged from cross-sectional studies showing a link between increased serum ferritin concentrations and the $\operatorname{Met} S^{(53,66)}$. A recent cross-sectional study on fifty-nine South Korean postmenopausal vegetarians and 48 age-matched postmenopausal non-vegetarian controls revealed that the prevalence of the MetS was 33.9 and $47.9 \%$, respectively ${ }^{(67)}$. Further, serum ferritin was positively correlated with FBG $(r 0 \cdot 264, P<0 \cdot 01)$, TAG $(r 0.232, P<0.05)$ and the NCEP criteria score for the MetS $(r 0.214, P<0 \cdot 05)$.

\section{Future research}

The current evidence that shows that an association between consumption of a vegetarian dietary pattern and a reduced prevalence or risk of the MetS comes from cross-sectional and case-control studies. There is a need for further research to be conducted, particularly prospective cohort studies to evaluate the effect of vegetarian dietary patterns on reducing the incidence of the MetS, and clinical trials should be designed to explore vegetarian dietary patterns for the reversal of the MetS in high-risk populations. This research could contribute to reduce the societal and economic burdens associated with the disorder.

\section{Acknowledgements}

The authors' contributions are as follows: J. S. conceived the manuscript; J. S. and M. W. wrote the manuscript; J. S. and M. W. approved the final manuscript.

The authors have no conflicts of interest.

\section{References}

1. Heidemann C, Schulze MB, Franco OH, et al. (2008) Dietary patterns and risk of mortality from cardiovascular disease, cancer, and all causes in a prospective cohort of women. Circulation 118, 230-237.

2. Paradis AM, Perusse L \& Vohl MC (2006) Dietary patterns and associated lifestyles in individuals with and without familial history of obesity: a cross-sectional study. Int J Behav Nutr Phys Act 3, 38.

3. Pryer JA, Cook A \& Shetty P (2001) Identification of groups who report similar patterns of diet among a representative national sample of British adults aged 65 years of age or more. Public Health Nutr 4, 787-795.

4. Rumawas ME, Meigs JB, Dwyer JT, et al. (2009) Mediterranean-style dietary pattern, reduced risk of metabolic syndrome traits, and incidence in the Framingham Offspring Cohort. Am J Clin Nutr 90, 1608-1614.

5. Ambrosini GL, Huang RC, Mori TA, et al. (2010) Dietary patterns and markers for the metabolic syndrome in Australian adolescents. Nutr Metab Cardiovasc Dis 20, 274-283.

6. McNeill AM, Rosamond WD, Girman CJ, et al. (2005) The metabolic syndrome and 11-year risk of incident cardiovascular disease in the atherosclerosis risk in communities study. Diabetes Care 28, 385-390.

7. Grundy SM (2006) Metabolic syndrome: connecting and reconciling cardiovascular and diabetes worlds. J Am Coll Cardiol 47, 1093-1100.

8. Beltran-Sanchez H, Harhay MO, Harhay MM, et al. (2013) Prevalence and trends of metabolic syndrome in the adult US population, 1999-2010. J Am Coll Cardiol 62, 697-703.

9. Misra A \& Khurana L (2009) The metabolic syndrome in South Asians: epidemiology, determinants, and prevention. Metab Syndr Relat Disord 7, 497-514.

10. Boudreau DM, Malone DC, Raebel MA, et al. (2009) Health care utilization and costs by metabolic syndrome risk factors. Metab Syndr Relat Disord 7, 305-314.

11. Grundy SM, Cleeman JI, Daniels SR, et al. (2005) Diagnosis and management of the metabolic syndrome: an American Heart Association/National Heart, Lung, and Blood Institute scientific statement: Executive Summary. Crit Pathw Cardiol 4, 198-203.

12. Zimmet P, Magliano D, Matsuzawa Y, et al. (2005) The metabolic syndrome: a global public health problem and a new definition. J Atheroscler Thromb 12, 295-300.

13. Fraser GE (1999) Associations between diet and cancer, ischemic heart disease, and all-cause mortality in non-Hispanic white California Seventh-day Adventists. Am J Clin Nutr 70, 532S-538S.

14. Key TJ, Fraser GE, Thorogood M, et al. (1999) Mortality in vegetarians and nonvegetarians: detailed findings from a collaborative analysis of 5 prospective studies. Am J Clin Nutr 70, 516S-524S.

15. Bamia C, Trichopoulos D, Ferrari P, et al. (2007) Dietary patterns and survival of older Europeans: the EPIC-Elderly Study (European Prospective Investigation into Cancer and Nutrition). Public Health Nutr 10, 590-598.

16. Fung TT, van Dam RM, Hankinson SE, et al. (2010) Low-carbohydrate diets and all-cause and cause-specific mortality: two cohort studies. Ann Intern Med 153, 289-298.

17. Knoops KT, Groot de LC, Fidanza F, et al. (2006) Comparison of three different dietary scores in relation to 10-year mortality in elderly European subjects: the HALE project. Eur J Clin Nutr 60, 746-755.

18. Sofi F, Abbate R, Gensini GF, et al. (2010) Accruing evidence on benefits of adherence to the Mediterranean diet on 
health: an updated systematic review and meta-analysis. Am J Clin Nutr 92, 1189-1196.

19. Trichopoulou A, Bamia C \& Trichopoulos D (2009) Anatomy of health effects of Mediterranean diet: Greek EPIC prospective cohort study. BMJ 338, b2337.

20. Trichopoulou A, Costacou T, Bamia C, et al. (2003) Adherence to a Mediterranean diet and survival in a Greek population. NEJM 348, 2599-2608.

21. Bazelmans C, De Henauw S, Matthys C, et al. (2006) Healthy food and nutrient index and all cause mortality. Eur $J$ Epidemiol 21, 145-152.

22. Waijers PM, Ocke MC, van Rossum CT, et al. (2006) Dietary patterns and survival in older Dutch women. Am J Clin Nutr 83, $1170-1176$.

23. Fraser GE (2003) Diet and the risk of coronary heart disease. In Diet, Life Expectancy, and Chronic Disease, pp. 59-84 [GE Fraser, editor]. New York: Oxford University Press.

24. Tonstad S, Butler T, Yan R, et al. (2009) Type of vegetarian diet, body weight, and prevalence of type 2 diabetes. Diabetes Care 32, 791-796.

25. Tonstad S, Stewart K, Oda K, et al. (2013) Vegetarian diets and incidence of diabetes in the Adventist Health Study-2. Nutr Metab Cardiovasc Dis 23, 292-299.

26. Appleby PN, Davey GK \& Key TJ (2002) Hypertension and blood pressure among meat eaters, fish eaters, vegetarians and vegans in EPIC-Oxford. Public Health Nutr 5, 645-654.

27. Pettersen BJ, Anousheh R, Fan J, et al. (2012) Vegetarian diets and blood pressure among white subjects: results from the Adventist Health Study-2 (AHS-2). Public Health Nutr 15, 1909-1916.

28. Tantamango-Bartley Y, Jaceldo-Siegl K, Fan J, et al. (2013) Vegetarian diets and the incidence of cancer in a low-risk population. Cancer Epidemiol Biomarkers Prev 22, 286-294.

29. Orlich MJ, Singh PN, Sabaté J, et al. (2013) Vegetarian dietary patterns and mortality in Adventist Health Study 2. JAMA 173, 1230-1238.

30. Sabaté J \& Blix G (2001) Vegetarian diets and obesity prevention. In Vegetarian Nutrition, pp. 91-107 [J Sabaté, editor]. Boca Raton, FL: CRC Press.

31. Chiang JK, Lin YL, Chen CL, et al. (2013) Reduced risk for metabolic syndrome and insulin resistance associated with ovo-lacto-vegetarian behavior in female Buddhists: a casecontrol study. PloS ONE 8, e71799.

32. Valachovicová M, Krajcovicova-Kudlackova M, Blazicek P, et al. (2006) No evidence of insulin resistance in normal weight vegetarians. A case control study. Eur J Nutr 45, 52-54.

33. Weaver CM (2009) Should dairy be recommended as part of a healthy vegetarian diet? Point. Am J Clin Nutr 89, 1634S-1637s.

34. Pereira MA, Jacobs DR Jr, Van Horn L, et al. (2002) Dairy consumption, obesity, and the insulin resistance syndrome in young adults: the CARDIA Study. JAMA 287, 2081-2089.

35. Rizzo NS, Sabaté J, Jaceldo-Siegl K, et al. (2011) Vegetarian dietary patterns are associated with a lower risk of metabolic syndrome: the adventist health study 2. Diabetes Care 34, 1225-1227.

36. Yang SY, Li XJ, Zhang W, et al. (2012) Chinese lactovegetarian diet exerts favorable effects on metabolic parameters, intima-media thickness, and cardiovascular risks in healthy men. Nutr Clin Pract 27, 392-398.

37. Amini M, Esmaillzadeh A, Shafaeizadeh S, et al. (2010) Relationship between major dietary patterns and metabolic syndrome among individuals with impaired glucose tolerance. Nutrition 26, 986-992.
38. Gadgil MD, Anderson CA, Kandula NR, et al. (2013) Dietary patterns in Asian Indians in the United States: an analysis of the metabolic syndrome and atherosclerosis in South Asians living in America Study. J Acad Nutr Diet 114, 238-243.

39. Shang P, Shu Z, Wang Y, et al. (2011) Veganism does not reduce the risk of the metabolic syndrome in a Taiwanese cohort. Asia Pac J Clin Nutr 20, 404-410.

40. Ashen MD \& Blumenthal RS (2005) Clinical practice. Low HDL cholesterol levels. NEJM 353, 1252-1260.

41. Meksawan K, Pendergast DR, Leddy JJ, et al. (2004) Effect of low and high fat diets on nutrient intakes and selected cardiovascular risk factors in sedentary men and women. J Am Coll Nutr 23, 131-140.

42. Barnard ND, Cohen J, Jenkins DJ, et al. (2006) A low-fat vegan diet improves glycemic control and cardiovascular risk factors in a randomized clinical trial in individuals with type 2 diabetes. Diabetes Care 29, 1777-1783.

43. Hung CJ, Huang PC, Li YH, et al. (2006) Taiwanese vegetarians have higher insulin sensitivity than omnivores. Br J Nutr 95, 129-135.

44. Jenkins DJ, Kendall CW, Marchie A, et al. (2003) Type 2 diabetes and the vegetarian diet. Am J Clin Nutr 78, 610S-616S.

45. Kuo CS, Lai NS, Ho LT, et al. (2004) Insulin sensitivity in Chinese ovo-lactovegetarians compared with omnivores. Eur J Clin Nutr 58, 312-316.

46. Lovejoy JC, Windhauser MM, Rood JC, et al. (1998) Effect of a controlled high-fat versus low-fat diet on insulin sensitivity and leptin levels in African-American and Caucasian women. Metabolism 47, 1520-1524.

47. Baxter AJ, Coyne T \& McClintock C (2006) Dietary patterns and metabolic syndrome - a review of epidemiologic evidence. Asia Pac J Clin Nutr 15, 134-142.

48. Williams DE, Prevost AT, Whichelow MJ, et al. (2000) A cross-sectional study of dietary patterns with glucose intolerance and other features of the metabolic syndrome. Br J Nutr 83, 257-266.

49. Wirfalt E, Hedblad B, Gullberg B, et al. (2001) Food patterns and components of the metabolic syndrome in men and women: a cross-sectional study within the Malmo Diet and Cancer cohort. Am J Epidemiol 154, 1150-1159.

50. Lutsey PL, Steffen LM \& Stevens J (2008) Dietary intake and the development of the metabolic syndrome: the Atherosclerosis Risk in Communities study. Circulation 117, 754-761.

51. Garduno-Diaz SD \& Khokhar S (2013) South Asian dietary patterns and their association with risk factors for the metabolic syndrome. J Hum Nutr Diet 26, 145-155.

52. Huang CJ, Fan YC, Liu JF, et al. (2011) Characteristics and nutrient intake of Taiwanese elderly vegetarians: evidence from a national survey. BrJ Nutr 106, 451-460.

53. Kim J \& Jo I (2011) Grains, vegetables, and fish dietary pattern is inversely associated with the risk of metabolic syndrome in South korean adults. J Am Diet Assoc 111, 1141-1149.

54. Jaaskelainen P, Magnussen CG, Pahkala K, et al. (2012) Childhood nutrition in predicting metabolic syndrome in adults: the cardiovascular risk in Young Finns Study. Diabetes Care 35, 1937-1943.

55. Matthews VL, Wien M \& Sabaté J (2011) The risk of child and adolescent overweight is related to types of food consumed. Nutr J 10, 71.

56. Kastorini CM, Milonis HJ, Esposito K, et al. (2011) The effect of Mediterranean diet on metabolic syndrome and its components: a meta-analysis of 50 studies and 534,906 individuals. J Am Coll Cardiol 57, 1299-1313. 
57. Babio N, Bullo M, Basora J, et al. (2009) Adherence to the Mediterranean diet and risk of metabolic syndrome and its components. Nutr Metab Cardiovasc Dis 19, 563-570.

58. Salas-Salvado J, Fernandez-Ballart J, Ros E, et al. (2008) Effect of a Mediterranean diet supplemented with nuts on metabolic syndrome status: one-year results of the PREDIMED randomized trial. Arch Intern Med 168, 2449-2458.

59. Kendall CW, Josse AR, Esfahani A, et al. (2010) Nuts, metabolic syndrome and diabetes. Br J Nutr 104, 465-473.

60. Jaceldo-Siegl K, Haddad E, Oda K, et al. (2014) Tree nuts are inversely associated with metabolic syndrome and obesity: the Adventist health study-2. PloS ONE 9, e85133.

61. O'Neil CE, Keast DR, Nicklas TA, et al. (2012) Out-of-hand nut consumption is associated with improved nutrient intake and health risk markers in US children and adults: National Health and Nutrition Examination Survey 1999-2004. Nutr Res 32, 185-194.
62. Esmaillzadeh A, Kimiagar M, Mehrabi Y, et al. (2006) Fruit and vegetable intakes, C-reactive protein, and the metabolic syndrome. Am J Clin Nutr 84, 1489-1497.

63. Haddad EH, Berk LS, Kettering JD, et al. (1999) Dietary intake and biochemical, hematologic, and immune status of vegans compared with nonvegetarians. Am J Clin Nutr 70, 586S-593S.

64. Pongstaporn W \& Bunyaratavej A (1999) Hematological parameters, ferritin and vitamin $\mathrm{B}_{12}$ in vegetarians. $J$ Med Assoc Thai 82, 304-311.

65. Wilson AK \& Ball MJ (1999) Nutrient intake and iron status of Australian male vegetarians. Eur J Clin Nutr 53, 189-194.

66. Sun L, Franco OH, Hu FB, et al. (2008) Ferritin concentrations, metabolic syndrome, and type 2 diabetes in middle-aged and elderly chinese. J Clin Endocrinol Metab 93, 4690-4696.

67. Kim MH \& Bae YJ (2012) Postmenopausal vegetarians' low serum ferritin level may reduce the risk for metabolic syndrome. Biol Trace Elem Res 149, 34-41. 